\title{
The Solvability of a Class of General Nonlinear Implicit Variational Inequalities Based on Perturbed Three-Step Iterative Processes with Errors
}

\author{
Zeqing Liu, ${ }^{1}$ Shin Min Kang, ${ }^{2}$ and Jeong Sheok Ume ${ }^{3}$ \\ ${ }^{1}$ Department of Mathematics, Liaoning Normal University, P.O. Box 200, Dalian, \\ Liaoning 116029, China \\ ${ }^{2}$ Department of Mathematics and the Research Institute of Natural Science, \\ Gyeongsang National University, Jinju 660-701, South Korea \\ ${ }^{3}$ Department of Applied Mathematics, Changwon National University, Changwon 641-733, South Korea
}

Correspondence should be addressed to Shin Min Kang, smkang@nongae.gsnu.ac.kr

Received 23 October 2007; Accepted 25 January 2008

Recommended by Mohammed Khamsi

We introduce and study a new class of general nonlinear implicit variational inequalities, which includes several classes of variational inequalities and variational inclusions as special cases. By applying the resolvent operator technique and fixed point theorem, we suggest a new perturbed three-step iterative algorithm with errors for solving the class of variational inequalities. Several existence and uniqueness results of solutions for the general nonlinear implicit variational inequalities, and convergence and stability results of the sequence generated by the algorithm are obtained. The results presented in this paper extend, improve, and unify a host of results in recent literatures.

Copyright $(2008$ Zeqing Liu et al. This is an open access article distributed under the Creative Commons Attribution License, which permits unrestricted use, distribution, and reproduction in any medium, provided the original work is properly cited.

\section{Introduction}

In recent years, various extensions and generalizations of the variational inequalities have been considered and studied. For details, we refer to [1-33], and the references therein. It is well known that one of the most interesting and important problems in the variational inequality theory is the development of an efficient iterative algorithm to compute approximate solutions of various variational inequalities and inclusions. In 1994, Hassouni and Moudafi [8] introduced a perturbed algorithm for solving a class of variatioanl inclusions. In 2003, Fang and Huang [7] introduced the definitions of $H$-monotone operator and its resolvent operator, established the Lipschitz continuity of the resolvent operator, constructed an iterative 
algorithm, and obtained the existence of solutions for a class of variational inclusions and convergence of the iterative algorithm. In 2004, Liu and Kang [19] established several existence and uniqueness theorems and convergence and stability results of perturbed three-step iterative algorithm with errors for a class of completely generalized nonlinear quasivariational inequalities.

Inspired and motivated by the recent research works in [1-28], in this paper, we introduce and study a new class of general nonlinear implicit variational inequalities, which includes the variational inequalities and variational inclusions in [1-28] as special cases. By applying the resolvent operator technique and fixed point theorem, we suggest a new perturbed three-step iterative process with errors for solving the general nonlinear implicit variational inequalities. Several existence and uniqueness results of solutions for the general nonlinear implicit variational inequalities involving $H$-monotone, strongly monotone, relaxed monotone, relaxed Lipschitz and generalized pseudocontractive operators, and convergence and stability results of the perturbed three-step iterative process with errors are given. The results presented in this paper extend, improve, and unify a host of results in recent literatures.

\section{Preliminaries}

Throughout this paper, we assume that $X$ is a real Hilbert space endowed with a norm $\|\cdot\|$ and an inner product $\langle\cdot, \cdot\rangle$, respectively, $2^{X}$ stands for the family of all the nonempty subsets of $X$, and $I$ denotes the identity operator on $X$. Assume that $H, g, m, A, B, C, D, E: X \rightarrow X$ and $N, M: X \times X \rightarrow X$ are operators, and $W: X \times X \rightarrow 2^{X}$ is a multivalued operator. Given $f \in X$, we consider the following problem: find $u \in X$ such that

$$
f \in N(A(u), B(u))-M(C(u), D(u))+W((g-m)(u), E(u)),
$$

which is called the general nonlinear implicit variational inequality, where $(g-m)(x)=g(x)-m(x)$ for all $x \in X$.

Some special cases of problem (2.1) are as follows.

(A) If $f=M=0, E=I$, then problem (2.1) reduces to the following problem: find $u \in H$ such that

$$
0 \in N(A(u), B(u))+W((g-m)(u), u),
$$

which is called the completely generalized strongly nonlinear implicit quasivariational inclusion in [20].

(B) If $f=0, E=I, N(x, y)=M(x, y)=x$ for any $x, y \in X$, then problem (2.1) is equivalent to finding $u \in X$ such that

$$
0 \in A(u)-C(u)+W((g-m)(u), u),
$$

which is called the generalized nonlinear implicit quasivariational inclusion in [10].

(C) If $f=0, N(x, y)=M(x, y)=x$, and $W(x, y)=W(x)$ for any $x, y, z \in X$, then problem (2.1) collapses to seeking $u \in X$ such that

$$
0 \in A(u)-C(u)+W((g-m)(u)),
$$

which is called the generalized equation by Uko [23]. 
(D) If $f=M=0, g-m=I, N(x, y)=x$, and $W(x, y)=W(x)$ for any $x, y \in X$, then problem (2.1) is equivalent to finding $u \in X$ such that

$$
0 \in A(u)+W(u)
$$

which was introduced and studied by Fang and Huang [7].

For appropriate and suitable choices of the operators $H, g, m, A, B, C, D, E, N, M, W$ and the element $f$, one can obtain various classes of variational inequalities and variational inclusions in [1-33] as special cases of problem (2.1).

We now recall and introduce the following definitions and results.

Definition 2.1. Let $N: X \times X \rightarrow X, g, b, c, H: X \rightarrow X$ be operators and let $W: X \rightarrow 2^{X}$ be a multivalued operator.

(a1) $g$ is said to be Lipschitz continuous and strongly monotone if there exist positive constants $s$ and $t$ satisfying, respectively,

$$
\|g(x)-g(y)\| \leq s\|x-y\|, \quad\langle g(x)-g(y), x-y\rangle \geq t\|x-y\|^{2}, \quad \forall x, y \in X
$$

(a2) $W$ is said to be maximal monotone if $W$ is monotone and $(I+\rho W)(X)=X$ for any $\rho>0$;

(a3) $W$ is said to be $H$-monotone if $W$ is monotone and $(H+\rho W)(X)=X$ for any $\rho>0$;

(a4) $b$ is called strongly monotone with respect to $H$ and the first argument of $N$ if there exists a positive constant $s$ satisfying

$$
\langle N(b(x), u)-N(b(y), u), H(x)-H(y)\rangle \geq s\|x-y\|^{2}, \quad \forall x, y, u \in X ;
$$

(a5) $b$ is called relaxed Lipschitz with respect to $H$ and the first argument of $N$ if there exists a positive constant $s$ satisfying

$$
\langle N(b(x), u)-N(b(y), u), H(x)-H(y)\rangle \leq-s\|x-y\|^{2}, \quad \forall x, y, u \in X
$$

(a6) $b$ is called relaxed monotone with respect to $H$ and the second argument of $N$ if there exists a positive constant $s$ satisfying

$$
\langle N(u, b(x))-N(u, b(y)), H(x)-H(y)\rangle \geq-s\|x-y\|^{2}, \quad \forall x, y \in X ;
$$

(a7) $b$ is called generalized pseudocontractive with respect to $g$ if there exists a positive constant $s$ satisfying

$$
\langle b(x)-b(y), g(x)-g(y)\rangle \leq s\|x-y\|^{2}, \quad \forall x, y \in X
$$

(a8) $N$ is called Lipschitz continuous with respect to the first argument if there exists a positive constant $s$ satisfying

$$
\|N(x, u)-N(y, u)\| \leq s\|x-y\|, \quad \forall x, y \in X .
$$


Similarly, we can define the Lipschitz continuity of $N$ with respect to the second argument. On the other hand, if $N(x, y)=x$ for any $x, y \in X$, then Definition 2.1 reduces to the usual concepts of strong monotonicity, relaxed monotonicity, and Lipschitz continuity. It is known that a maximal monotone operator need not be $H$-monotone for some $H$, and if $W$ is $H$-monotone and $H$ is strictly monotone, then $W$ is maximal monotone.

Definition 2.2 (see [7]). Let $H: X \rightarrow X$ be a strictly monotone operator and let $W: X \rightarrow 2^{X}$ be an $H$-monotone operator. For any given $\rho>0$, the resolvent operator $R_{W, \rho}^{H}: X \rightarrow X$ is defined by

$$
R_{W, \rho}^{H}(x)=(H+\rho W)^{-1}(x), \quad \forall x \in X
$$

Definition 2.3 (see [34]). Let $g: X \rightarrow X$ be an operator and $x_{0} \in X$. Assume that $x_{n+1}=f\left(g, x_{n}\right)$ define an iteration procedure which yields a sequence of points $\left\{x_{n}\right\}_{n \geq 0}$ in $X$. Suppose that $F(g)=\{x \in X: x=g(x)\} \neq \varnothing$ and $\left\{x_{n}\right\}_{n \geq 0}$ converges to some $u \in F(g)$. Let $\left\{z_{n}\right\}_{n \geq 0} \subset X$ and $\epsilon_{n}=\left\|z_{n+1}-f\left(g, z_{n}\right)\right\|$ for all $n \geq 0$. Iflim $\lim _{n \rightarrow \infty} \epsilon_{n}=0$ implies that $\lim _{n \rightarrow \infty} z_{n}=u$, then the iteration procedure defined by $x_{n+1}=f\left(g, x_{n}\right)$ is said to be $g$-stable or stable with respect to $g$.

Lemma 2.4 (see [35]). Let $\left\{a_{n}\right\}_{n \geq 0},\left\{b_{n}\right\}_{n \geq 0}$, and $\left\{c_{n}\right\}_{n \geq 0}$ be nonnegative sequences satisfying

$$
a_{n+1} \leq\left(1-t_{n}\right) a_{n}+t_{n} b_{n}+c_{n}, \quad \forall n \geq 0,
$$

where $\left\{t_{n}\right\}_{n \geq 0} \subset[0,1], \sum_{n=0}^{\infty} t_{n}=\infty, \lim _{n \rightarrow \infty} b_{n}=0$, and $\sum_{n=0}^{\infty} c_{n}<\infty$. Then $\lim _{n \rightarrow \infty} a_{n}=0$.

Lemma 2.5 (see [7]). Let $H: X \rightarrow X$ be a strongly monotone operator with constant $r$ and let $W: X \rightarrow 2^{X}$ be an H-monotone operator. Then the resolvent operator $R_{W, \rho}^{H}: X \rightarrow X$ is Lipschitz continuous with constant $r^{-1}$.

\section{Existence, convergence, and stability}

Now, we use the resolvent operator technique to establish the equivalence between the general nonlinear implicit variational inequality (2.1) and the fixed point problem.

Lemma 3.1. Let $\lambda$ and $\rho$ be two positive constants, let $H: X \rightarrow X$ be a strictly monotone operator, let $W: X \times X \rightarrow 2^{X}$ be a multivalued operator such that for any fixed $x \in X, W(\cdot, E(x))$ is $H$-monotone, and

$$
Y(x)=H((g-m)(x))-\rho N(A(x), B(x))+\rho M(C(x), D(x))+\rho f, \quad \forall x \in X,
$$

where $H, g, m, A, B, C, D, E: X \rightarrow X$ and $N, M: X \times X \rightarrow X$ are operators. Then the following statements are equivalent:

(b1) the general nonlinear implicit variational inequality (2.1) possesses a solutio $u \in X$;

(b2) there exists $u \in X$ satisfying

$$
g(u)=m(u)+R_{W(\cdot, E(u)), \rho}^{H}(Y(u))
$$


(b3) the mapping $G: X \rightarrow X$ defined by

$$
G(x)=(1-\lambda) x+\lambda\left[x-(g-m)(x)+R_{W(\cdot, E(x)), \rho}^{H}(Y(x))\right], \quad \forall x \in X
$$

has a fixed point $u \in X$.

Proof. It is clear that (b1) holds if and only if $Y(u) \in(H+\rho W(\cdot, E(u)))((g-m)(u))$, which is equivalent to (3.2) by the definition of the resolvent operator. On the other hand, (3.3) means that $G$ has a fixed point $u \in X$ if and only if (3.2) holds. This completes the proof.

Remark 3.2. Lemma 3.1 extends and improves Lemma 3.1 in $[1,7,10,12,19-22,32]$, Theorem 3.2 in [6], Lemma 3.2 in [25], Theorem 2.1 in [8, 24, 26], and Lemma 2.2 [27].

Based on Lemma 3.1, we suggest the following perturbed three-step iterative process with errors for the general nonlinear implicit variational inequality (2.1).

Algorithm 3.3. Let $A, B, C, D, E, g, m, H, H_{n}: X \rightarrow X, N, M: X \times X \rightarrow X$ be operators, $W, W_{n}$ : $X \times X \rightarrow 2^{X}$ satisfy that for any $x \in X, W(\cdot, E(x))$ is $H$-monotone and $W_{n}(\cdot, E(x))$ is $H_{n}$-monotone for each $n \geq 0$. Given $f, u_{0} \in X$, the iterative sequence $\left\{u_{n}\right\}_{n \geq 0}$ is defined by

$$
\begin{aligned}
w_{n} & =\left(1-c_{n}\right) u_{n}+c_{n}\left[u_{n}-(g-m)\left(u_{n}\right)+R_{W_{n}\left(\cdot, E\left(u_{n}\right)\right), \rho}^{H_{n}}\left(Y\left(u_{n}\right)\right)\right]+r_{n}, \\
v_{n} & =\left(1-b_{n}\right) u_{n}+b_{n}\left[w_{n}-g\left(w_{n}\right)+m\left(w_{n}\right)+R_{W_{n}\left(\cdot, E\left(w_{n}\right)\right), \rho}^{H_{n}}\left(Y\left(w_{n}\right)\right)\right]+q_{n}, \\
u_{n+1} & =\left(1-a_{n}\right) u_{n}+a_{n}\left[v_{n}-(g-m)\left(v_{n}\right)+R_{W_{n}\left(\cdot, E\left(v_{n}\right)\right), \rho}^{H_{n}}\left(Y\left(v_{n}\right)\right)\right]+p_{n}, \quad n \geq 0,
\end{aligned}
$$

where $Y$ is defined by (3.1), $\left\{p_{n}\right\}_{n \geq 0},\left\{q_{n}\right\}_{n \geq 0}$, and $\left\{r_{n}\right\}_{n \geq 0}$ are sequences in $X$ introduced to take into account possible in inexact computation, and the sequences $\left\{a_{n}\right\}_{n \geq 0},\left\{b_{n}\right\}_{n \geq 0}$, and $\left\{c_{n}\right\}_{n \geq 0}$ are sequences in [0.1] satisfying

$$
\sum_{n=0}^{\infty} a_{n}=\infty, \quad \sum_{n=0}^{\infty}\left\|p_{n}\right\|<\infty, \quad \lim _{n \rightarrow \infty}\left\|q_{n}\right\|=\lim _{n \rightarrow \infty} b_{n}\left\|r_{n}\right\|=0 .
$$

Remark 3.4. Algorithm 3.1 in [1, 7, 12, 19, 21, 25, 32], Algorithm 2.1 in [8, 27], and Algorithm 5.1 in $[9,11]$, the Ishikawa-type perturbed iterative algorithm in [10], the Ishikawa-type perturbed iterative algorithm with errors in [20], Algorithms 3.1 and 3.2 in [22] are special cases of Algorithm 3.3 in this paper.

Next, we study those conditions under which the approximate solutions $u_{n}$ obtained from Algorithm 3.3 converge strongly to the unique solution $u \in X$ of the general nonlinear implicit variational inequality (2.1), and the convergence, under suitable conditions, is stable.

Theorem 3.5. Let $H: X \rightarrow X$ be strongly monotone and Lipschitz continuous with constants $s$ and $h$, respectively. Let $H_{n}: X \rightarrow X$ be strongly monotone with constant $s_{n}$ for each $n \geq 0$ and let $g: X \rightarrow X$ be Lipschtiz continuous and strongly monotone with constants $t$ and $p$, respectively. Assume that $m, A, B, C, D, E,: X \rightarrow X$ are Lipschitz continuous with constants $q, a, b, c, d$, and $e$, respectively. Let $W, W_{n}: X \times X \rightarrow 2^{X}$ satisfy that for each $x \in X, W(\cdot, E(x))$ is H-monotone and $W_{n}(\cdot, E(x))$ is $H_{n}$-monotone for each $n \geq 0$. Let $N: X \times X \rightarrow X$ be Lipschitz continuous with constants $i$ and $j$ with respect to the first and second arguments, respectively. Let $M: X \times X \rightarrow X$ be Lipschitz continuous with constants $k$ and $l$ with respect to the first and second arguments, respectively. Suppose that $A$ is strongly monotone with constant $\alpha$ with respect to $H(g-m)$ and the first argument of $N, C$ is relaxed Lipschitz with constant $\gamma$ with respect to $H(g-m)$ and the first argument of $M$, and $D$ is relaxed 
monotone with constant $\delta$ with respect to $H(g-m)$ and the second argument of $M$. Let

$$
\begin{aligned}
& P=\sqrt{1-2 p+t^{2}}+q+\eta e, \quad J=i^{2} a^{2}-T^{2}, \\
& T=j b+\sqrt{h^{2}(t+q)^{2}-2 \gamma+k^{2} c^{2}}+\sqrt{h^{2}(t+q)^{2}+2 \delta+l^{2} d^{2}}, \\
& K=\alpha-s(1-P) T, \quad L=h^{2}(t+q)^{2}-s^{2}(1-P)^{2}>0 .
\end{aligned}
$$

Let $\left\{x_{n}\right\}_{n \geq 0}$ be any sequence in $X$ and define $\left\{\epsilon_{n}\right\}_{n \geq 0} \subset[0, \infty)$ by

$$
\begin{aligned}
& \epsilon_{n}=\left\|x_{n+1}-\left\{\left(1-a_{n}\right) x_{n}+a_{n}\left[y_{n}-(g-m)\left(y_{n}\right)+R_{W_{n}\left(\cdot, E\left(y_{n}\right)\right), \rho}^{H_{n}}\left(Y\left(y_{n}\right)\right)\right]+p_{n}\right\}\right\|, \\
& y_{n}=\left(1-b_{n}\right) x_{n}+b_{n}\left[z_{n}-(g-m)\left(z_{n}\right)+R_{W_{n}\left(\cdot, E\left(z_{n}\right)\right), \rho}^{H_{n}}\left(Y\left(z_{n}\right)\right)\right]+q_{n}, \\
& z_{n}=\left(1-c_{n}\right) x_{n}+c_{n}\left[x_{n}-(g-m)\left(x_{n}\right)+R_{W_{n}\left(\cdot, E\left(x_{n}\right)\right), \rho}^{H_{n}}\left(Y\left(x_{n}\right)\right)\right]+r_{n}, \quad \forall n \geq 0,
\end{aligned}
$$

where $Y$ is defined by (3.1). If there exist positive constants $\rho, \eta$, and $\eta_{n}$ satisfying

$$
\begin{gathered}
\left\|R_{W(\cdot, x), \rho}^{H}(z)-R_{W(\cdot, y), \rho}^{H}(z)\right\| \leq \eta\|x-y\|, \quad \forall x, y, z \in X, \\
\left\|R_{W_{n}(\cdot, x), \rho}^{H_{n}}(z)-R_{W_{n}(\cdot, y), \rho}^{H_{n}}(z)\right\| \leq \eta_{n}\|x-y\|, \quad \forall x, y, z \in X, n \geq 0, \\
\lim _{n \rightarrow \infty}\left\|R_{W_{n}(\cdot, E(x)), \rho}^{H_{n}}(Y(x))-R_{W(\cdot, E(x)), \rho}^{H}(Y(x))\right\|=0, \quad \forall x \in X, \\
\lim _{n \rightarrow \infty} \eta_{n}=\eta, \quad \lim _{n \rightarrow \infty} s_{n}=s, \\
P+s^{-1} \rho T<1,
\end{gathered}
$$

and one of the following conditions:

$$
\begin{gathered}
\left|\rho-K J^{-1}\right|<J^{-1} \sqrt{K^{2}-L J}, \quad J>0,|K|>\sqrt{L J} \\
\left|\rho-K J^{-1}\right|>-J^{-1} \sqrt{K^{2}-L J}, \quad J<0
\end{gathered}
$$

then for any given $f \in X$, the general nonlinear implicit variational inequality (2.1) has a unique solution $u \in X$ and the sequence $\left\{u_{n}\right\}_{n \geq 0}$ defined by Algorithm 3.3 converges strongly to $u$. Moreover, if there exists a constant $\beta>0$ satisfying

$$
a_{n} \geq \beta, \quad \forall n \geq 0,
$$

then $\lim _{n \rightarrow \infty} x_{n}=u$ if and only if $\lim _{n \rightarrow \infty} \epsilon_{n}=0$.

Proof. First of all, we claim that the mapping $G$ defined by (3.3) has a unique fixed point $u \in X$, where $\lambda$ is a constant in $(0,1]$. Let $x, y$ be two arbitrary elements in $X$. Note that $g$ is Lipschtiz continuous and strongly monotone with constants $t$ and $p$, respectively. It follows that

$$
\|x-y-[g(x)-g(y)]\| \leq \sqrt{1-2 p+t^{2}}\|x-y\|
$$


Since $A$ is strongly monotone with constant $\alpha$ with respect to $H(g-m)$ and the first argument of $N, C$ is relaxed Lipschitz with constant $\gamma$ with respect to $H(g-m)$ and the first argument of $M$, and $D$ is relaxed monotone with constant $\delta$ with respect to $H(g-m)$ and the second argument of $M$, it follows from the Lipschitz continuity of $A, B, C, D$, and $H$, and the Lipschitz continuity of $N$ and $M$ with respect to the first and second arguments, respectively, that

$$
\begin{aligned}
& \|y(x)-y(y)\| \\
& \leq\|H((g-m)(x))-H((g-m)(y))-\rho[N(A(x), B(x))-N(A(y), B(x))]\| \\
& +\rho\|N(A(y), B(x))-N(A(y), B(y))\| \\
& +\rho\|H((g-m)(x))-H((g-m)(y))+M(C(x), D(x))-M(C(y), D(x))\| \\
& +\rho\|H((g-m)(x))-H((g-m)(y))-M(C(y), D(x))+M(C(y), D(y))\| \\
& \leq\left[\|H((g-m)(x))-H((g-m)(y))\|^{2}\right. \\
& \quad-2 \rho\langle N(A(x), B(x))-N(A(y), B(x)), H((g-m)(x))-H((g-m)(y))\rangle \\
& \left.\quad+\rho^{2}\|N(A(x), B(x))-N(A(y), B(x))\|^{2}\right]^{1 / 2}+\rho j b\|x-y\| \\
& +\rho\left[\|H((g-m)(x))-H((g-m)(y))\|^{2}\right. \\
& \quad+2\langle M(C(x), D(x))-M(C(y), D(x)), H((g-m)(x))-H((g-m)(y))\rangle \\
& \left.\quad+\|M(C(x), D(x))-M(C(y), D(x))\|^{2}\right]^{1 / 2} \\
& +\rho\left[\|H((g-m)(x))-H((g-m)(y))\|^{2}\right. \\
& \quad-2\langle M(C(y), D(x))-M(C(y), D(y)), H((g-m)(x))-H((g-m)(y))\rangle \\
& \left.\quad+\|M(C(y), D(x))-M(C(y), D(y))\|^{2}\right]^{1 / 2} \\
& \leq\left[\sqrt{h^{2}(t+q)^{2}-2 \alpha \rho+\rho^{2} i^{2} a^{2}}+\rho T\right]\|x-y\| .
\end{aligned}
$$

In view of Lemma 2.5, (3.3), (3.6), (3.8), (3.16), and (3.17), we deduce that

$$
\begin{aligned}
&\|G(x)-G(y)\| \\
& \leq(1-\lambda)\|x-y\|+\lambda\|x-y-(g-m)(x)+(g-m)(y)\|+\lambda\left\|R_{W(\cdot, E(x)), \rho}^{H}(Y(x))-R_{W(\cdot, E(y)), \rho}^{H}(Y(y))\right\| \\
& \leq {\left[1-\lambda\left(1-\sqrt{1-2 p+t^{2}}-q\right)\right]\|x-y\|+\lambda\left\|R_{W(\cdot, E(x)), \rho}^{H}(Y(x))-R_{W(\cdot, E(y)), \rho}^{H}(Y(x))\right\| } \\
&+\lambda\left\|R_{W(\cdot, E(x)), \rho}^{H}(Y(x))-R_{W(\cdot, E(y)), \rho}^{H}(Y(y))\right\| \\
& \leq {\left[1-\lambda\left(1-\sqrt{1-2 p+t^{2}}-q-\eta e\right)\right]\|x-y\|+\lambda s^{-1}\|Y(x)-Y(y)\| } \\
& \leq(1-\lambda(1-\theta))\|x-y\|,
\end{aligned}
$$

where

$$
\theta=P+s^{-1}\left[\sqrt{h^{2}(t+q)^{2}-2 \rho \alpha+\rho^{2} i^{2} a^{2}}+\rho T\right]>0 .
$$


In light of (3.6), (3.12), and (3.19), we derive that

$$
\theta<1 \Longleftrightarrow \sqrt{h^{2}(t+q)^{2}-2 \rho \alpha+\rho^{2} i^{2} a^{2}}<s(1-P)-\rho T \Longleftrightarrow J \rho^{2}-2 K \rho<-L .
$$

It follows from one of (3.13) and (3.14) that

$$
\theta<1 \text {. }
$$

Thus (3.18) implies that $G$ is a contraction mapping, and hence $G$ has a unique fixed point $u \in X$. By Lemma 3.1, we conclude that the general nonlinear implicit variational inequality (2.1) possesses a unique solution $u \in X$ and

$$
\begin{aligned}
u & =\left(1-c_{n}\right) u+c_{n}\left[u-(g-m)(u)+R_{W(\cdot, E(u)), \rho}^{H}(Y(u))\right] \\
& =\left(1-b_{n}\right) u+b_{n}\left[u-(g-m)(u)+R_{W(\cdot E(u)), \rho}^{H}(Y(u))\right] \\
& =\left(1-a_{n}\right) u+a_{n}\left[u-(g-m)(u)+R_{W(\cdot, E(u)), \rho}^{H}(Y(u))\right], \quad \forall n \geq 0 .
\end{aligned}
$$

Next, we prove that $\lim _{n \rightarrow \infty} u_{n}=u$. Set

$$
\begin{aligned}
& \theta_{n}=P_{n}+s_{n}^{-1}\left[\sqrt{h^{2}(t+q)^{2}-2 \rho \alpha+\rho^{2} i^{2} a^{2}}+\rho T\right], \\
& P_{n}=\sqrt{1-2 p+t^{2}}+q+e \eta_{n}, \\
& g_{n}=\left\|R_{W_{n}(\cdot, E(u)), \rho}^{H_{n}}(Y(u))-R_{W(\cdot, E(u)), \rho}^{H}(Y(u))\right\|, \quad \forall n \geq 0 .
\end{aligned}
$$

In terms of (3.11), (3.19), and (3.21), we know that $\lim _{n \rightarrow \infty} \theta_{n}=\theta<1$. Hence there exists some positive integer $Q$ satisfying

$$
\theta_{n}<\frac{1}{2}(1+\theta)<1, \quad \forall n \geq Q
$$

Using Lemma 2.5, Algorithm 3.3, (3.22), and (3.24), we know that for $n>Q$,

$$
\begin{aligned}
& \left\|w_{n}-u\right\| \\
& \begin{aligned}
\leq & \left(1-c_{n}\right)\left\|u_{n}-u\right\|+c_{n}\left[\left\|u_{n}-u-(g-m)\left(u_{n}\right)+(g-m)(u)\right\|\right. \\
& \left.+\left\|R_{W_{n}\left(\cdot, E\left(u_{n}\right)\right), \rho}^{H_{n}}\left(Y\left(u_{n}\right)\right)-R_{W(\cdot, E(u)), \rho}^{H}(Y(u))\right\|\right]+\left\|r_{n}\right\| \\
\leq & {\left[1-c_{n}\left(1-\sqrt{1-2 p+t^{2}}-q\right)\right]\left\|u_{n}-u\right\| } \\
& +c_{n}\left[\left\|R_{W_{n}\left(\cdot, E\left(u_{n}\right)\right), \rho}^{H_{n}}\left(Y\left(u_{n}\right)\right)-R_{W_{n}\left(\cdot, E\left(u_{n}\right)\right), \rho}^{H_{n}}(Y(u))\right\|+\left\|R_{W_{n}\left(\cdot, E\left(u_{n}\right)\right), \rho}^{H_{n}}\left(Y\left(u_{n}\right)\right)-R_{W_{n}(\cdot, E(u)), \rho}^{H_{n}}(Y(u))\right\|\right. \\
& \left.\quad+\left\|R_{W_{n}(\cdot, E(u)), \rho}^{H_{n}}(Y(u))-R_{W(\cdot, E(u)), \rho}^{H}(Y(u))\right\|\right]+\left\|r_{n}\right\|
\end{aligned}
\end{aligned}
$$


Zeqing Liu et al.

$$
\begin{aligned}
& \leq\left[1-c_{n}\left(1-\sqrt{1-2 p+t^{2}}-q\right)\right]\left\|u_{n}-u\right\| \\
& \quad+c_{n}\left[s_{n}^{-1}\left\|Y\left(u_{n}\right)-Y(u)\right\|+\eta_{n}\left\|E\left(u_{n}\right)-E(u)\right\|+g_{n}\right]+\left\|r_{n}\right\| \\
& \leq\left[1-c_{n}\left(1-\sqrt{1-2 p+t^{2}}-q\right)\right]\left\|u_{n}-u\right\| \\
& \quad+c_{n}\left\{s _ { n } ^ { - 1 } \left[\| H\left((g-m)\left(u_{n}\right)\right)\right.\right. \\
& \quad-H((g-m)(u))-\rho\left[N\left(A\left(u_{n}\right), B\left(u_{n}\right)\right)-N\left(A(u), B\left(u_{n}\right)\right)\right] \| \\
& \quad+\rho\left\|N\left(A(u), B\left(u_{n}\right)\right)-N(A(u), B(u))\right\| \\
& \quad+\rho\left\|H\left((g-m)\left(u_{n}\right)\right)-H((g-m)(u))+M\left(C\left(u_{n}\right), D\left(u_{n}\right)\right)-M\left(C(u), D\left(u_{n}\right)\right)\right\| \\
& \left.\quad+\rho\left\|H\left((g-m)\left(u_{n}\right)\right)-H((g-m)(u))-M\left(C(u), D\left(u_{n}\right)\right)+M(C(u), D(u))\right\|\right] \\
& \left.\quad+e \eta_{n}\left\|u_{n}-u\right\|+g_{n}\right\}+\left\|r_{n}\right\| \\
& \leq\left(1-c_{n}\right)\left\|u_{n}-u\right\|+c_{n} \theta_{n}\left\|u_{n}-u\right\|+c_{n} g_{n}+\left\|r_{n}\right\| \\
& \leq\left\|u_{n}-u\right\|+c_{n} g_{n}+\left\|r_{n}\right\| .
\end{aligned}
$$

Similarly, we conclude that

$$
\begin{aligned}
\left\|v_{n}-u\right\| & \leq\left(1-b_{n}\right)\left\|u_{n}-u\right\|+b_{n} \theta_{n}\left\|w_{n}-u\right\|+b_{n} g_{n}+\left\|q_{n}\right\| \\
& \leq\left\|u_{n}-u\right\|+b_{n}\left(2 g_{n}+\left\|r_{n}\right\|\right)+\left\|q_{n}\right\|, \\
\left\|u_{n+1}-u\right\| & \leq\left(1-a_{n}\right)\left\|u_{n}-u\right\|+a_{n} \theta_{n}\left\|v_{n}-u\right\|+a_{n} g_{n}+\left\|p_{n}\right\| \\
& \leq\left[1-\left(1-\theta_{n}\right) a_{n}\right]\left\|u_{n}-u\right\|+a_{n}\left(3 g_{n}+\left\|q_{n}\right\|+b_{n}\left\|r_{n}\right\|\right)+\left\|p_{n}\right\| \\
& \leq\left[1-\frac{1}{2}(1-\theta) a_{n}\right]\left\|u_{n}-u\right\|+a_{n}\left(3 g_{n}+\left\|q_{n}\right\|+b_{n}\left\|r_{n}\right\|\right)+\left\|p_{n}\right\|
\end{aligned}
$$

for $n>Q$. It is easy to see that $\lim _{n \rightarrow \infty}\left\|u_{n}-u\right\|=0$ by Lemma 2.4, (3.5), (3.10), and (3.27).

Assume that (3.15) holds. As in the proof of (3.27), we easily deduce that

$$
\begin{aligned}
\|(1- & \left.a_{n}\right) x_{n}+a_{n}\left[y_{n}-(g-m)\left(y_{n}\right)+R_{W_{n}\left(\cdot, E\left(y_{n}\right)\right), \rho}^{H_{n}}\left(Y\left(y_{n}\right)\right)\right]+p_{n}-u \| \\
& \leq\left(1-\left(1-\theta_{n}\right) a_{n}\right)\left\|x_{n}-u\right\|+a_{n}\left(3 g_{n}+\left\|q_{n}\right\|+b_{n}\left\|r_{n}\right\|\right)+\left\|p_{n}\right\| \\
& \leq\left(1-\frac{1}{2}(1-\theta) \beta\right)\left\|x_{n}-u\right\|+3 g_{n}+\left\|q_{n}\right\|+b_{n}\left\|r_{n}\right\|+\left\|p_{n}\right\|
\end{aligned}
$$

for $n>Q$.

Suppose that $\lim _{n \rightarrow \infty} x_{n}=u$. By virtue of (3.5), (3.7), (3.10), and (3.28), we see that

$$
\begin{aligned}
\epsilon_{n} & \leq\left\|x_{n+1}-u\right\|+\left\|\left(1-a_{n}\right) x_{n}+a_{n}\left[y_{n}-(g-m)\left(y_{n}\right)+R_{W_{n}\left(\cdot, E\left(y_{n}\right)\right), \rho}^{H_{n}}\left(Y\left(y_{n}\right)\right)\right]+p_{n}-u\right\| \\
& \leq\left\|x_{n+1}-u\right\|+\left(1-\frac{1}{2}(1-\theta) \beta\right)\left\|x_{n}-u\right\|+3 g_{n}+\left\|q_{n}\right\|+b_{n}\left\|r_{n}\right\|+\left\|p_{n}\right\| \longrightarrow 0
\end{aligned}
$$

as $n \rightarrow \infty$. Therefore, $\lim _{n \rightarrow \infty} \epsilon_{n}=0$. 
Conversely, suppose that $\lim _{n \rightarrow \infty} \epsilon=0$. It follows from (3.7), (3.22), and (3.28) that

$$
\begin{aligned}
& \left\|x_{n+1}-u\right\| \\
& \quad \leq\left\|\left(1-a_{n}\right) x_{n}+a_{n}\left[y_{n}-(g-m)\left(y_{n}\right)+R_{W_{n}\left(\cdot, E\left(y_{n}\right)\right), \rho}^{H_{n}}\left(Y\left(y_{n}\right)\right)\right]+p_{n}-u\right\|+\epsilon_{n} \\
& \quad \leq\left(1-\frac{1}{2}(1-\theta) \beta\right)\left\|x_{n}-u\right\|+3 g_{n}+\left\|q_{n}\right\|+b_{n}\left\|r_{n}\right\|+\left\|p_{n}\right\|+\epsilon_{n}
\end{aligned}
$$

for $n>Q$. Using (3.5), (3.10), (3.30), and Lemma 2.4, we infer that $\lim _{n \rightarrow \infty} x_{n}=u$. This completes the proof.

Theorem 3.6. Let $H, W,\left\{H_{n}\right\}_{n \geq 0},\left\{W_{n}\right\}_{n \geq 0}, g, A, B, C, D, E, J, T, L,\left\{x_{n}\right\}_{n \geq 0}$, and $\left\{\epsilon_{n}\right\}_{n \geq 0}$ be as in Theorem 3.5 and

$$
P=\sqrt{1-2(p-\epsilon)+q^{2}+t^{2}}+\eta e .
$$

Let $m: X \rightarrow X$ be generalized pseudocontractive with constant $\epsilon$ with respect to $(I-g)$ and be Lipschitz continuous with constant $q$. If there exist positive constants $\rho, \eta$, and $\eta_{n}$ satisfying (3.8)-(3.12) and one of (3.13) and (3.14), then for any given $f \in X$, the general nonlinear implicit variational inequality (2.1) has a unique solution $u \in X$ and the sequence $\left\{u_{n}\right\}_{n \geq 0}$ defined by Algorithm 3.3 converges strongly to $u$. Moreover, if (3.15) holds, then $\lim _{n \rightarrow \infty} x_{n}=u$ if and only if $\lim _{n \rightarrow \infty} \epsilon_{n}=0$.

Proof. Because $m$ is generalized pseudocontractive with constant $\epsilon$ with respect to $(I-g)$ and Lipschitz continuous with constant $q, g$ is Lipschtiz continuous and strongly monotone with constants $t$ and $p$, respectively, it follows that

$$
\begin{aligned}
\|(I- & g)(x)-(I-g)(y)+m(x)-m(y) \| \\
& =\left[\|m(x)-m(y)\|^{2}+2\langle m(x)-m(y),(I-g)(x)-(I-g)(y)\rangle+\|(I-g)(x)-(I-g)(y)\|^{2}\right]^{1 / 2} \\
& \leq\left[\left(q^{2}+2 \epsilon\right)\|x-y\|^{2}+\|x-y\|^{2}-2\langle g(x)-g(y), x-y\rangle+\|g(x)-g(y)\|^{2}\right]^{1 / 2} \\
& \leq \sqrt{1-2(p-\epsilon)+q^{2}+t^{2}}\|x-y\|, \quad \forall x, y \in X .
\end{aligned}
$$

The rest of the proof now follows that as in the proof of Theorem 3.5. This completes the proof.

Theorem 3.7. Let $H, W,\left\{H_{n}\right\}_{n \geq 0},\left\{W_{n}\right\}_{n \geq 0}, g, m, B, E, J, K,\left\{x_{n}\right\}_{n \geq 0}$, and $\left\{\epsilon_{n}\right\}_{n \geq 0}$ be as in Theorem 3.5, and

$$
\begin{aligned}
& P=\left(1+s^{-1}\right)\left(\sqrt{1-2 p+t^{2}}+q\right)+\eta e+s^{-1}(t+q) \sqrt{1-2 s+h^{2}}, \\
& T=j b+\sqrt{1+2 \delta+l^{2} d^{2}}+\sqrt{1-2 \gamma+k^{2} c^{2}}, \\
& L=1-s^{2}(1-P)^{2}>0 .
\end{aligned}
$$

Let $A: X \rightarrow X$ be Lipschitz continuous with constant $a$ and strongly monotone with constant $\alpha$ with respect to $I$ and the first argument of $N$. Let $C: X \rightarrow X$ be Lipschitz continuous with constant 
$c$ and relaxed Lipschitz with constant $\gamma$ with respect to $I$ and the first argument of $M$. Assume that $D: X \rightarrow X$ is Lipschitz continuous with constant $d$ and relaxed monotone with constant $\delta$ with respect to $I$ and the second argument of $M$. If there exist positive constants $\rho, \eta$, and $\eta_{n}$ satisfying (3.8)-(3.12) and one of (3.13) and (3.14), then for any given $f \in X$, the general nonlinear implicit variational inequality (2.1) has a unique solution $u \in X$ and the sequence $\left\{u_{n}\right\}_{n \geq 0}$ defined by Algorithm 3.3 converges strongly to $u$. Moreover, if (3.15) holds, then $\lim _{n \rightarrow \infty} x_{n}=u$ if and only if $\lim _{n \rightarrow \infty} \epsilon=0$.

Proof. Notice that

$$
\begin{aligned}
& \| H((g-m)(x))-H((g-m)(y))-\rho[N(A(x), B(x))-N(A(y), B(x))] \\
&-\rho[N(A(y), B(x))-N(A(y), B(y))]+\rho[M(C(x), D(x))-M(C(y), D(x))] \\
&+\rho[M(C(y), D(x))-M(C(y), D(y))] \| \\
& \leq\|H((g-m)(x))-H((g-m)(y))-(g-m)(x)+(g-m)(y)\| \\
&+\|(g-m)(x)-(g-m)(y)-x+y\| \\
&+\|x-y-\rho[N(A(x), B(x))-N(A(y), B(x))]\|+\rho j b \\
&+\rho\|x-y+M(C(x), D(x))-M(C(y), D(x))\| \\
&+\rho\|x-y-M(C(y), D(x))+M(C(y), D(y))\| \\
& \leq {\left[(t+q) \sqrt{1-2 s+h^{2}}+\sqrt{1-2 p+t^{2}}+q+\sqrt{1-2 \rho \alpha+\rho^{2} i^{2} a^{2}}+\rho T\right]\|x-y\| }
\end{aligned}
$$

for any $x, y \in X$. The rest of the proof is identical with the proof of Theorem 3.5. This completes the proof.

Following similar arguments as in the proof of Theorems 3.5, 3.6, and 3.7, we obtain immediately the result below:

Theorem 3.8. Let $H, W,\left\{H_{n}\right\}_{n \geq 0},\left\{W_{n}\right\}_{n \geq 0}, g, A, B, C, D, E, J, K, T, L,\left\{x_{n}\right\}_{n \geq 0}$, and $\left\{\epsilon_{n}\right\}_{n \geq 0}$ be as in Theorem 3.7, and $m$ be as in Theorem 3.6, and

$$
P=\left(1+s^{-1}\right) \sqrt{1-2(p-\epsilon)+q^{2}+t^{2}}+\eta e+s^{-1}(t+q) \sqrt{1-2 s+h^{2}}
$$

If there exist positive constants $\rho, \eta$, and $\eta_{n}$ satisfying (3.8)-(3.12) and one of (3.13) and (3.14), then for any given $f \in X$, the general nonlinear implicit variational inequality (2.1) has a unique solution $u \in X$ and the sequence $\left\{u_{n}\right\}_{n \geq 0}$ defined by Algorithm 3.3 converges strongly to $u$. Moreover, if (3.15) holds, then $\lim _{n \rightarrow \infty} x_{n}=u$ if and only if $\lim _{n \rightarrow \infty} \epsilon=0$.

Remark 3.9. Theorems 3.5-3.8 establish both the existence and uniqueness of solutions for the general nonlinear implicit variational inclusion (2.1) and show the convergence and stability of the perturbed three-step iterative process with errors under certain conditions. 
Remark 3.10. Theorems 3.5-3.8 extend, improve, and unify Theorem 3.4 in $[1,6]$, Theorem 2.1 in [8], Theorem 3.1 in [7, 12, 21, 22, 25, 32], Theorem 2.3 in [24], Theorem 2.2 in [26], Theorem $5.1[9,11]$, Theorem 4.1 in $[10,20]$, Theorems 4.1-4.3 in [19], Theorems 1 and 2 in [23], and Theorems $3.1-3.6$ in $[3,13]$.

\section{Acknowledgment}

This work was supported by the Science Research Foundation of Educational Department of Liaoning Province (20060467) and the Korea Research Foundation Grant funded by the Korean Government (MOEHRD, Basic Research Promotion Fund) (KRF-2006-312-C00026).

\section{References}

[1] S. Adly, "Perturbed algorithms and sensitivity analysis for a general class of variational inclusions," Journal of Mathematical Analysis and Applications, vol. 201, no. 2, pp. 609-630, 1996.

[2] C. Baiocchi and A. Capelo, Variational and Quasivariational Inequalities: Applications to Free Boundary Problems, A Wiley-Interscience Publication, John Wiley \& Sons, New York, NY, USA, 1984.

[3] Y. J. Cho, J. H. Kim, N.-J. Huang, and S. M. Kang, "Ishikawa and Mann iterative processes with errors for generalized strongly nonlinear implicit quasivariational inequalities," Publicationes Mathematicae Debrecen, vol. 58, no. 4, pp. 635-649, 2001.

[4] R. W. Cottle, F. Giannessi, and J. L. Lions, Variational Inequalities and Complementarity Problems, Theory and Applications, John Wiley \& Sons, New York, NY, USA, 1980.

[5] R. Glowinski, Numerical Methods for Nonlinear Variational Problems, Springer Series in Computational Physics, Springer, Berlin, Germany, 1984.

[6] J.-S. Guo and J. C. Yao, "Extension of strongly nonlinear quasivariational inequalities," Applied Mathematics Letters, vol. 5, no. 3, pp. 35-38, 1992.

[7] Y.-p. Fang and N.-J. Huang, " $H$-monotone operator and resolvent operator technique for variational inclusions," Applied Mathematics and Computation, vol. 145, no. 2-3, pp. 795-803, 2003.

[8] A. Hassouni and A. Moudafi, "A perturbed algorithm for variational inclusions," Journal of Mathematical Analysis and Applications, vol. 185, no. 3, pp. 706-712, 1994.

[9] N.-J. Huang, "On the generalized implicit quasivariational inequalities," Journal of Mathematical Analysis and Applications, vol. 216, no. 1, pp. 197-210, 1997.

[10] N.-J. Huang, "Mann and Ishikawa type perturbed iterative algorithms for generalized nonlinear implicit quasivariational inclusions," Computers \& Mathematics with Applications, vol. 35, no. 10, pp. $1-7,1998$.

[11] N.-J. Huang, M.-R. Bai, Y. J. Cho, and S. M. Kang, "Generalized nonlinear mixed quasivariational inequalities," Computers \& Mathematics with Applications, vol. 40, no. 2-3, pp. 205-215, 2000.

[12] N.-J. Huang, Y.-P. Fang, and Y. J. Cho, "Perturbed three-step approximation processes with errors for a class of general implicit variational inclusions," Journal of Nonlinear and Convex Analysis, vol. 4, no. 2, pp. 301-308, 2003.

[13] J. K. Kim, Y. Y. Tang, and N.-J. Huang, "Ishikawa and Mann iterative processes with errors for generalized strongly nonlinear quasivariational inequalities," Nonlinear Functional Analysis and Applications, vol. 4, no. 1, pp. 41-54, 1999.

[14] Z. Liu, J. S. Ume, and S. M. Kang, "General strongly nonlinear quasivariational inequalities with relaxed Lipschitz and relaxed monotone mappings," Journal of Optimization Theory and Applications, vol. 114, no. 3, pp. 639-656, 2002.

[15] Z. Liu, J. S. Ume, and S. M. Kang, "Resolvent equations technique for general variational inclusions," Proceedings of the Japan Academy Series A, vol. 78, no. 10, pp. 188-193, 2002.

[16] Z. Liu, L. Debnath, S. M. Kang, and J. S. Ume, "Sensitivity analysis for parametric completely generalized nonlinear implicit quasivariational inclusions," Journal of Mathematical Analysis and Applications, vol. 277, no. 1, pp. 142-154, 2003.

[17] Z. Liu and S. M. Kang, "Generalized multivalued nonlinear quasivariational inclusions," Mathematische Nachrichten, vol. 253, pp. 45-54, 2003. 
[18] Z. Liu, S. M. Kang, and J. S. Ume, "Completely generalized multivalued strongly quasivariational inequalities," Publicationes Mathematicae Debrecen, vol. 62, no. 1-2, pp. 187-204, 2003.

[19] Z. Liu and S. M. Kang, "Convergence and stability of perturbed three-step iterative algorithm for completely generalized nonlinear quasivariational inequalities," Applied Mathematics and Computation, vol. 149, no. 1, pp. 245-258, 2004.

[20] S. H. Shim, S. M. Kang, N.-J. Huang, and Y. J. Cho, "Perturbed iterative algorithms with errors for completely generalized strongly nonlinear implicit quasivariational inclusions," Journal of Inequalities and Applications, vol. 5, no. 4, pp. 381-395, 2000.

[21] A. H. Siddiqi and Q. H. Ansari, "Strongly nonlinear quasivariational inequalities," Journal of Mathematical Analysis and Applications, vol. 149, no. 2, pp. 444-450, 1990.

[22] A. H. Siddiqi and Q. H. Ansari, "General strongly nonlinear variational inequalities," Journal of Mathematical Analysis and Applications, vol. 166, no. 2, pp. 386-392, 1992.

[23] L. U. Uko, "Strongly nonlinear generalized equations," Journal of Mathematical Analysis and Applications, vol. 220, no. 1, pp. 65-76, 1998.

[24] R. U. Verma, "An iterative procedure for approximating fixed points of relaxed monotone operators," Numerical Functional Analysis and Optimization, vol. 17, no. 9-10, pp. 1045-1051, 1996.

[25] R. U. Verma, "Generalized variational inequalities and associated nonlinear equations," Czechoslovak Mathematical Journal, vol. 48, no. 3, pp. 413-418, 1998.

[26] R. U. Verma, "Generalized pseudo-contractions and nonlinear variational inequalities," Publicationes Mathematicae Debrecen, vol. 53, no. 1-2, pp. 23-28, 1998.

[27] R. U. Verma, "The solvability of a class of generalized nonlinear variational inequalities based on an iterative algorithm," Applied Mathematics Letters, vol. 12, no. 4, pp. 51-53, 1999.

[28] J. C. Yao, "Existence of generalized variational inequalities," Operations Research Letters, vol. 15, no. 1, pp. 35-40, 1994.

[29] J. C. Yao, "The generalized quasivariational inequality problem with applications," Journal of Mathematical Analysis and Applications, vol. 158, no. 1, pp. 139-160, 1991.

[30] J. C. Yao, "Multivalued variational inequalities with $K$-pseudomonotone operators," Journal of Optimization Theory and Applications, vol. 83, no. 2, pp. 391-403, 1994.

[31] J. C. Yao, "Abstract variational inequality problems and a basic theorem of complementarity," Computers \& Mathematics with Applications, vol. 25, no. 1, pp. 73-79, 1993.

[32] L. C. Zeng, "Iterative algorithms for finding approximate solutions for general strongly nonlinear variational inequalities," Journal of Mathematical Analysis and Applications, vol. 187, no. 2, pp. 352-360, 1994.

[33] L. C. Zeng, S. M. Guu, and J. C. Yao, "Iterative algorithm for completely generalized set-valued strongly nonlinear mixed variational-like inequalities," Computers $\mathcal{E}$ Mathematics with Applications, vol. 50, no. 5-6, pp. 935-945, 2005.

[34] A. M. Harder and T. L. Hicks, "Stability results for fixed point iteration procedures," Mathematica Japonica, vol. 33, no. 5, pp. 693-706, 1988.

[35] L. S. Liu, "Ishikawa and Mann iterative process with errors for nonlinear strongly accretive mappings in Banach spaces," Journal of Mathematical Analysis and Applications, vol. 194, no. 1, pp. 114-125, 1995. 